Cerebrovasc Dis 2008;26:570

DOI: $\underline{10.1159 / 000164559}$

\section{Reply to the Letter by Morelli et al: Vertebrobasilar Recording with Contrast Transcranial Doppler in Right-to-Left Shunt Diagnosis}

K.J. Daly, S. Hutchinson, C. McCollum

Department of Vascular Surgery, South Manchester University Hospital, Manchester, UK

We thank Morelli et al. [1] for their interest in our paper on peripheral artery detection of a venous-to-arterial circulation shunt [2].

The use of an occipital window is not a technique that we have tried when investigating shunts. We found that there was too much interference when we tried to use two $2-\mathrm{MHz}$ probes to insonate the middle cerebral artery and the common carotid artery. This could be a problem with insonation of the basilar artery, and we wonder whether Morelli et al. [1] used two probes with different frequencies. We had considerable difficulty in holding the probe on the common carotid, especially during provocation with coughing and the Valsalva maneuver. This may be easier to overcome with the occipital window, as presumably it is easier to hold the probe more firmly against the skull than to the front of the neck.
The relatively low sensitivity for detection of shunts (75\%) may limit the application of basilar artery insonation. However, the high sensitivity for the detection of medium to large shunts may indicate that this technique can reliably detect clinically significant shunts. We would still advocate the use of the common femoral artery when a temporal window is absent, as this is a simple sensitive alternative with a high positive predictive value of $92.3 \%$ for detection of a patent foramen ovale [2].

References

1 Morelli N, Gallerini S, Cafforio G, Chiti A, Baldacci F, Mancuso M, Orlandi G, Tartaglione A, Murri L: Vertebrobasilar recording with contrast transcranial doppler in right-to-left shunt diagnosis. Cerebrovasc $\mathrm{Dis} 2008 ; 26: 569$.

-2 Daly KJ, Hutchinson S, McCollum CN: Can a peripheral artery be used to detect venous to arterial circulation shunts? Cerebrovasc Dis 2007; 25:111-114

Kevin J. Daly

Department of Vascular Surgery

South Manchester University Hospital

Southmoor road, Manchester M23 9LT (UK)

Tel. +44 161291 5853, Fax +44 1612915854

E-Mail kjdaly@ukonline.co.uk

\title{
Erratum
}

In the article 'Acetylsalicylic Acid and Acetaminophen to Combat Elevated Body Temperature in Acute Ischemic Stroke', published in Cerebrovasc Dis 2004;17:118-122, the fourth author name was erroneously misprinted. The correct spelling is Gert Jan Luijckx. 